\title{
Evaluation of Pharmacologic Strategies for Prevention of Venous Thromboembolism in Elective Orthopedic
}

\section{Surgery}

\section{Running title: Pharmacologic Strategies for Prevention of VTE in Orthopedic Surgery}

\author{
Eleanor K. Broadbent ${ }^{1}$, Andrea Lewin ${ }^{1}$, Katelyn W. Sylvester ${ }^{1}$, Betsy Whitfield ${ }^{2}$ and Danielle Knowles ${ }^{1}$
}

1. Department of Pharmacy Services, Brigham and Women's Hospital, Boston, Massachusetts 02115, USA

2. Northeastern University, Boston, Massachusetts 02115, USA

\begin{abstract}
Background: Venous thromboembolism (VTE) is a common postoperative complication in orthopedic surgery and warrants the use of pharmacologic thromboprophylaxis. The purpose of this study was to analyze current prescribing practices at our institution and to assess outcomes of pharmacologic strategies used for VTE prophylaxis following major orthopedic surgery. Methods: This was a single center, retrospective analysis at a tertiary academic medical center. Patients were included if they were admitted for total hip or knee arthroplasties and received pharmacologic thromboprophylaxis (warfarin INR goal 1.8-2.3, aspirin at variable doses, low molecular weight heparin (LMWH), DOAC) post-operatively from January 2017 to June 2017. Patients were excluded if they had a history of VTE or were on long-term anticoagulation. Major endpoints included incidence of thrombotic and bleeding events within 30 days post-surgery. Results: A total of 476 patients were screened; 89 were excluded and 387 were included $($ warfarin $=183$, aspirin $=$ 201 , LMWH $=2$, apixaban =1). Two patients in the warfarin group experienced a deep vein thrombosis compared with one patient in the aspirin group $(p=0.51)$. Major bleeding occurred in one patient in the warfarin group and none in the aspirin group. Total bleeding rates occurred in $2.7 \%$ and $1.0 \%$ in the warfarin and aspirin groups, respectively. Conclusion: There has been an increase in the use of aspirin at our facility in postoperative orthopedic surgery patients without an increase in DOAC use. No difference was detected in safety and efficacy endpoints in our warfarin versus aspirin patients.
\end{abstract}

Key words: Venous thromboembolism, prophylaxis, orthopedic surgery, aspirin, warfarin.

\section{Introduction}

Venous thromboembolism (VTE) is a common postoperative complication after orthopedic surgery and warrants the use of prophylaxis for thrombosis prevention. Orthopedic patients are at higher risk for developing VTE as a result of exposure to all pathophysiologic processes that comprise Virchow's triad [1]. Venous blood stasis develops from the use of a tourniquet, bed rest, and immobilization, while endothelial vascular injuries can arise from surgical manipulations of the limb. Trauma during surgery can

Corresponding author: Eleanor Broadbent, PharmD, ebroadb1@ fairview.org, research field: infectious diseases. elevate thromboplastin levels and the use of polymethylmethacrylate bone cement has the potential to increase hypercoagulability. Previous studies demonstrated an incidence rate of symptomatic VTE of $15 \%$ to $30 \%$ in post-operative orthopedic surgery patients who were not given prophylaxis [2]. With the introduction of thromboprophylaxis in these patients, the risk of VTE has decreased to $1 \%$ to $2 \%$. Several different prophylactic strategies varying by agent, intensity, and duration are approved for use in patients who undergo total hip arthroplasties (THA) and total knee arthroplasties (TKA). Pharmacologic thromboprophylaxis agents used in this population include aspirin, warfarin, low-molecular weight 
heparins (LMWHs) such as enoxaparin and dalteparin, and fondaparinux. Three direct oral anticoagulants (rivaroxaban, apixaban, and dabigatran) have also been approved for this indication.

In 2010, our anticoagulation management service (AMS) evaluated different pharmacologic strategies for VTE prophylaxis following major orthopedic surgery at our institution [3]. Of the 462 patients who met inclusion criteria, 10 patients who received LMWH were excluded from the analysis due to low enrollment numbers of patients using that prophylaxis strategy. The analysis included 452 patients who underwent THA or TKA using different post-operative VTE prophylaxis strategies (warfarin INR goal 1.5-2.5, warfarin INR goal 1.8-2.3, and aspirin at variable doses). The number of patients in each respective group was 190, 214 and 48. This study found no difference in the incidence of VTE or bleeding 30-days post-surgery between the different strategies. Since this study, there have been updates to two sets of major guidelines. The 9th edition of the American College of Chest Physicians (ACCP) guidelines recognized aspirin, warfarin, LMWH, unfractionated heparin (UFH), fondaparinux, apixaban, rivaroxaban, and dabigatran for a minimum of 10-14 days as appropriate prophylactic approaches for the prevention of VTE after orthopedic surgery [2]. The 2011 American Academy of Orthopedic Surgeons (AAOS) guidelines give a moderate recommendation for the use of pharmacologic and/or mechanical prophylaxis in patients who are not at elevated risk for VTE or bleeding [4]. Recommendations for the use of a specific pharmacologic agent or duration are not addressed.

With the FDA approval of rivaroxaban, apixaban, and dabigatran for VTE prophylaxis in post-operative orthopedic surgery patients, there has been a growing body of literature evaluating the efficacy and safety of direct oral anticoagulants (DOACs) in this patient population [5-12]. After the guideline updates and approval of the DOACs, this retrospective cohort analysis sought to re-assess current prescribing practices at our institution and to assess outcomes of pharmacologic strategies used for VTE prophylaxis following THA and TKA.

\section{Material and Methods}

This was a single-center, retrospective, observational cohort analysis conducted at a tertiary-care teaching hospital affiliated with Harvard Medical School in Boston, Massachusetts. The study was approved by the Partners Healthcare Institutional Review Board.

Institutional operating room reports were utilized to create a list of consecutive patients who underwent THA or TKA surgeries between January 1, 2017 and June 30, 2017. Patients $\geq 18$ years of age who underwent THA or TKA at our institution and received pharmacologic thromboprophylaxis were included in the study. Risk of VTE was assessed during the initial pre-operative visit with the orthopedics team to determine which pharmacologic agent to use, however the specific choice of agent was provider-specific. Patients were excluded if they had a history of VTE, required long-term anticoagulation for another indication, were switched to a different agent during the prophylactic course of therapy, or received an abbreviated course of enoxaparin as a bridge to a second THA or TKA surgery.

Clinical data collected including baseline characteristics and concurrent medications are listed in Table 1. Antithrombotic strategies used for VTE prophylaxis after orthopedic surgery included warfarin (goal INR 1.8-2.3), LMWH, fondaparinux, aspirin (either $81 \mathrm{mg}$ or $325 \mathrm{mg}$ twice daily), and the DOAC agents, based on surgeon preference. In the warfarin group, INRs were drawn daily while inpatient, the day after discharge, and then twice weekly.

The major efficacy endpoint was the composite incidence of any thrombotic event defined as documentation of deep vein thrombosis (DVT) or pulmonary embolism (PE), myocardial infarction (MI), 
Table 1 Baseline characteristics.

\begin{tabular}{|c|c|c|c|}
\hline Characteristic & $\begin{array}{l}\text { Warfarin } \\
(n=183)\end{array}$ & $\begin{array}{l}\text { Aspirin } \\
(n=201)\end{array}$ & $p$-value \\
\hline Age (years), mean \pm SD & $63.3 \pm 14.6$ & $64.1 \pm 11.2$ & 0.09 \\
\hline Male, $n(\%)$ & $51(27.8)$ & $68(33.8)$ & 0.41 \\
\hline $\mathrm{BMI}$, mean $\pm \mathrm{SD}$ & $30.2 \pm 5.8$ & $28.4 \pm 5.8$ & $<0.05$ \\
\hline TKA, $n(\%)$ & $62(33.9)$ & $129(64.2)$ & $<0.05$ \\
\hline THA, $n(\%)$ & $121(66.1)$ & $72(35.8)$ & $<0.05$ \\
\hline Liver dysfunction, $n(\%)$ & $4(2.2)$ & $6(3.0)$ & 0.62 \\
\hline History of bleeding, $n(\%)$ & $16(8.7)$ & $16(8.0)$ & 0.78 \\
\hline Obesity, $n(\%)$ & $87(47.5)$ & $90(44.8)$ & 0.59 \\
\hline Alcohol use, $n(\%)$ & $64(35.0)$ & $126(62.7)$ & $<0.05$ \\
\hline Alcohol use estimation (drinks/week), mean \pm SD & $4.7 \pm 4.5$ & $3.4 \pm 4$ & 0.45 \\
\hline Current smoker, $n(\%)$ & $16(8.7)$ & $12(6.0)$ & 0.29 \\
\hline Active malignancy, $n(\%)$ & $0(0)$ & $4(2.0)$ & - \\
\hline Mechanical heart valve, $n(\%)$ & $0(0)$ & $0(0)$ & - \\
\hline Atrial fibrillation, $n(\%)$ & $0(0)$ & $6(3.0)$ & - \\
\hline Hyperthyroidism, $n(\%)$ & $0(0)$ & $2(1.0)$ & - \\
\hline GERD/PUD, $n(\%)$ & $56(30.6)$ & $74(36.8)$ & 0.19 \\
\hline Thrombocytosis, $n(\%)$ & $0(0)$ & $1(0.5)$ & - \\
\hline Anemia, $n(\%)$ & $48(26.2)$ & $20(1.0)$ & $<0.05$ \\
\hline Thrombocytopenia, $n(\%)$ & $8(4.4)$ & $4(2.0)$ & 0.18 \\
\hline $\mathrm{CHF}, n(\%)$ & $2(1.1)$ & $4(2.0)$ & 0.48 \\
\hline Hypothyroidism, $n(\%)$ & $20(10.9)$ & $30(14.9)$ & 0.09 \\
\hline HIT, $n(\%)$ & $0(0)$ & $1(0.5)$ & - \\
\hline
\end{tabular}

BMI-body mass index; TKA — total knee arthroplasty; THA—-total knee arthroplasty; GERD—gastroesophageal reflux disease; PUD — peptic ulcer disease; CHF — congestive heart failure; HIT — heparin-induced thrombocytopenia.

transient ischemic attack (TIA), or cerebrovascular accident (CVA) occurring within 30 days post-surgery. DVT and PE were diagnostically confirmed utilizing ultrasound and pulmonary angiography or other imaging studies, respectively. The major safety endpoint was a composite of International Society of Thrombosis and Haemostasis (ISTH)-defined major bleeding or minor bleeding throughout the duration of prophylaxis. Minor bleeding was considered any non-major bleeding event.

Secondary outcomes included treatment duration, hospital length of stay, time to reach therapeutic INR range, and percent of critical INRs for patients treated with warfarin. A critical INR was defined as an INR less than 1.5 or greater than 4 .

Patients were assessed throughout the duration of prophylaxis for safety endpoints and within 30-day post-surgery for efficacy endpoints, as available in electronic medical records. Patients were classified into the aspirin, warfarin, DOAC, or LMWH groups based on the clinician choice of prophylaxis. Baseline patient characteristics and treatment parameters were compared between treatment groups using the Student's $t$-test or Mann-Witney U test for continuous variables and the Chi-squared test for categorical variables. Statistical significance was set at a level of $p$ $<0.05$. Statistical analyses were performed using Social Science Statistics software (2018 version).

\section{Results}

A total of 476 patients who underwent major orthopedic surgery and received pharmacologic thromboprophylaxis were screened for inclusion. Of those, 89 patients were excluded (history of VTE $=52$; chronic anticoagulation $=34$; received enoxaparin bridge to second TKA or THA within three weeks of 
first surgery $=2$, switched prophylactic agents $=1$ ). Of the 387 patients $(81.3 \%)$ who met inclusion criteria, $183(47.3 \%)$ received warfarin, 201 (51.9\%) received aspirin, $2(0.5 \%)$ received $\mathrm{LMWH}$, and $1(0.3 \%)$ received apixaban. All patients treated with warfarin prophylaxis had doses titrated to an INR of 1.8-2.3 for a duration of three to four weeks, and patients in the aspirin group were dosed with either $81 \mathrm{mg} \operatorname{BID}(n=$ $48)$ or $325 \mathrm{mg}$ BID $(n=153)$. Only the warfarin and aspirin groups were included in the statistical analysis due to the low usage of LMWHs and DOACs. There were some differences in baseline characteristics and comorbidities noted between the warfarin and aspirin groups (Table 1). Warfarin patients had a higher BMI and a higher percentage of patients undergoing THA. The most common comorbidities observed among all patients were obesity (44.8\%), gastrointestinal reflux disease (33.9\%), hypothyroidism (13.9\%), and anemia $(17.7 \%)$. More patients in the aspirin group were alcohol users, while patients in the warfarin group had a higher incidence of anemia. The most common concomitant medications which may increase bleeding risk in both groups were levothyroxine, statins, and proton pump inhibitors. There was a significantly higher percentage of patients taking antibiotics and selective serotonin reuptake inhibitors or serotonin norepinephrine reuptake inhibitors at some time during their prophylaxis course in the aspirin group (Table 2).

No difference in the composite thrombotic event endpoint $(1.1 \%$ vs. $0.5 \% ; p=0.51)$ was detected between the warfarin and aspirin groups, respectively (Table 3). All thrombotic events were non-fatal, distal DVTs. There was no difference in the composite bleeding endpoint $(2.7 \%$ vs. $1.0 \% ; p=0.20)$ between the warfarin and aspirin groups, respectively. There was one major bleeding event in the warfarin group which was a gastrointestinal bleed resulting in a $\mathrm{Hgb}$ decrease of $>2$ $\mathrm{g} / \mathrm{dL}$. In the warfarin group 4 patients experienced a minor bleeding event compared to 2 patients in the aspirin group. Minor bleeding events included a hematoma and hemarthrosis event in the aspirin group and one rectal bleeding event, two hematoma reports, and one hematuria event in the warfarin group.

The mean treatment duration was 21.5 (SD 2.5) days in the warfarin group and 26.2 (SD 4.3) days in the aspirin group $(p<0.05)$. Median hospital length of stay was 3 (IQR 2-4) days in the warfarin group and 2 (IQR $1-3)$ days in the aspirin group $(p<0.05)$. Of the 1,390 INR values collected for patients in the warfarin group, 639 (46\%) were deemed critical, however, these included critically low INR values that were recorded prior to the first in-range INR (Table 3). The median time to therapeutic range for patients in the warfarin group was 6 days (IQR 5-7 days).

Table 2 Concomitant medications.

\begin{tabular}{|c|c|c|c|}
\hline Characteristic & $\begin{array}{l}\text { Warfarin } \\
(n=183)\end{array}$ & $\begin{array}{l}\text { Aspirin } \\
(n=201)\end{array}$ & $p$-value \\
\hline APAP, $n(\%)$ & $181(89.9)$ & $200(99.5)$ & 0.51 \\
\hline ASA, $n(\%)$ & $70(38.3)$ & - & - \\
\hline Clopidogrel, $n(\%)$ & $3(1.6)$ & $0(0)$ & - \\
\hline LMWH/UFH, $n(\%)$ & $5(2.7)$ & $0(0)$ & - \\
\hline NSAID, $n(\%)$ & $59(32.2)$ & $83(41.3)$ & 0.07 \\
\hline Antibiotics, $n(\%)$ & $13(7.1)$ & $30(14.9)$ & $<0.05$ \\
\hline Amiodarone, $n(\%)$ & $0(0)$ & $0(0)$ & - \\
\hline Levothyroxine, $n(\%)$ & $23(12.6)$ & $32(15.9)$ & 0.35 \\
\hline HMG-CoA reductase inhibitor, $n(\%)$ & $64(35.0)$ & $76(37.8)$ & 0.56 \\
\hline SSRI/SNRI, $n(\%)$ & $15(8.2)$ & $36(17.9)$ & $<0.05$ \\
\hline PPI, $n(\%)$ & $57(31.1)$ & $60(29.9)$ & 0.78 \\
\hline
\end{tabular}


Table 3 Major and minor endpoints.

\begin{tabular}{|c|c|c|c|}
\hline Endpoint & $\begin{array}{l}\text { Warfarin } \\
(n=183)\end{array}$ & $\begin{array}{l}\text { Aspirin } \\
(n=201)\end{array}$ & $p$-value \\
\hline \multicolumn{4}{|l|}{ Major endpoints } \\
\hline DVT, $n(\%)$ & $2(1.1)$ & $1(0.5)$ & 0.51 \\
\hline Total bleeding, $n(\%)$ & $5(2.7)$ & $2(1)$ & 0.2 \\
\hline Major bleeding, $n(\%)$ & $1(0.5)$ & $0(0)$ & - \\
\hline Minor bleeding, $n(\%)$ & $4(2.2)$ & $2(1)$ & 0.35 \\
\hline \multicolumn{4}{|l|}{ Minor endpoints } \\
\hline Treatment duration, mean \pm SD & $21.5 \pm 2.5$ & $26.2(4.3)$ & $<0.05$ \\
\hline Hospital length of stay, median (IQR) & $3(2-4)$ & $2(1-3)$ & $<0.05$ \\
\hline Critical INRs, $n$ (\% of total INRs collected) & $639(46)$ & - & - \\
\hline INRs $<1.5, n$ (\% of critical INRs collected $)$ & $610(95.5)$ & - & - \\
\hline INRs $>4, n$ ( $\%$ of critical INRs collected $)$ & $29(4.5)$ & - & - \\
\hline Time to therapeutic INR range, median (IQR) & $6(5-7)$ & - & \\
\hline
\end{tabular}

DVT — deep vein thrombosis; INR — international normalized ratio; IQR —interquartile range; SD—standard deviation.

\section{Discussion}

This analysis observed similar rates of thrombotic and bleeding events among patients who received aspirin compared with patients who received warfarin for VTE prophylaxis following orthopedic surgery. Higher doses of aspirin did not correlate with an increased risk of bleeding. The enrollment numbers in the DOAC and LMWH groups were too small to be assessed. Although not statistically significant, rates of VTE and of major and minor bleeding were numerically more frequent in the warfarin group. VTE event rates observed were less frequent compared to other studies, which may have been a result of excluding patients with a prior history of VTE [3, 8-13]. Bleeding event rates were also lower than those found in other studies, although this was likely due to the smaller enrollment numbers in this study [3, 8-13]. A larger cohort of patients would need to be analyzed to detect any significant differences in safety and efficacy outcomes. Hospital length of stay was significantly longer while treatment duration was statistically shorter in the warfarin group.

The 2010 study conducted at our institution analyzed data from 462 patients who underwent THA or TKA using different post-operative VTE prophylaxis strategies (warfarin INR goal 1.5-2.5, warfarin INR goal 1.8-2.3, and aspirin at variable doses) [3]. Of these three strategies, two primary events occurred in each warfarin group and there were no events in patients treated with aspirin. Rates of major and minor bleeding were $4.2 \%, 4.7 \%$, and $2.1 \%$ respectively. This study found no difference in the incidence of VTE or bleeding 30-days post-surgery. Compared to the current study, the enrollment numbers demonstrated an increase in aspirin prescribing post-operatively in our orthopedic surgery patient population $(10.4 \%$ vs. $51.9 \%$ ) [3].

The 9th edition of the ACCP guidelines equally recognize aspirin, warfarin, LMWH, low-dose UFH, fondaparinux, apixaban, rivaroxaban, and dabigatran (grade 1B recommendations) or intermittent pneumatic compression devices (grade $1 \mathrm{C}$ recommendation) as prophylactic approaches for the prevention of VTE after orthopedic surgery for a minimum of 10 to 14 days [2]. This update included the DOACs as an appropriate option for thromboprophylaxis and removed the recommended INR goal of 2-3 for patients treated with warfarin [14]. These guidelines do not recommend one option over another. The most recent edition of the AAOS guideline recommendations provided a moderate recommendation for the use of pharmacologic and/or mechanical prophylaxis in patients who are not at elevated risk for VTE or bleeding, without recommending the use of any specific agent [4]. The updates to the AAOS guidelines 
were more ambiguous and left the decision of what specific agent to use and the duration of therapy up to the clinician. The previous 2007 document specifically recommended LMWH, fondaparinux, warfarin, and aspirin [14]. Previously the ACCP recommended against aspirin for use in this population. The prescribing of aspirin and warfarin for VTE prophylaxis in the majority of TKA and THA patients at our institution correlates with the most recent editions of both the ACCP and AAOS guidelines.

Among our cohort, the very low enrollment of DOAC-treated patients was thought to be due to several factors. First, orthopedic surgeons at our institution have a long-standing relationship with our AMS for over 10 years. Providers may be less inclined to prescribe newer agents when their warfarin-treated patients are managed and followed closely by AMS. In addition, the lack of a commercially available reversal agent for apixaban and rivaroxaban during the study period may have dissuaded providers from prescribing DOACs. The prevention of bleeding around the time of surgery has been a major focus for practitioners at our institution and has historically contributed to the minimal prescribing of DOACs and LMWH in orthopedic patients. The approval of andexanet alfa, an FDA-approved recombinant modified human factor Xa decoy protein for factor-Xa inhibitor reversal in May 2018 could have a future impact on the prescribing of these newer agents [15]. Lastly, the relatively low cost of aspirin and warfarin compared to DOACs may have been a factor for prescribing those agents more frequently.

The patients enrolled in our cohort appear to have similar baseline characteristics as those enrolled in several trials which studied DOACs for post-operative orthopedic surgery VTE prophylaxis [9-12]. These similarities in age, sex, and BMI support the possibility of DOACs serving as a safe and effective alternative to warfarin and aspirin in our patient population. The question remains which prophylactic strategy should be used for any individual patient. Surgeon preference and patient VTE risk strongly influence agent selection at our institution.

Our study limitations include a small sample size from a single institution, which may increase the risk of a type 2 error and limit the generalizability of our results. The retrospective design of this analysis could have introduced the opportunity for selection bias. The small number of patients in the DOAC group and LMWH group did not allow for a meaningful assessment and comparison with the other strategies. In addition, outcomes associated with mechanical VTE prophylaxis or mobilization were not assessed.

\section{Conclusions}

There has been an increase in the use of aspirin at our facility in postoperative major orthopedic surgery patients without an increase in DOAC use. Among patients undergoing TKA or THA surgeries at our institution, there were no differences in the rates of thrombotic events or bleeding events between patients who received aspirin or warfarin for post-operative VTE prophylaxis. Warfarin patients had a longer hospital length of stay while the total duration of thromboprophylaxis was less compared to aspirin patients.

\section{References}

[1] Flevas, D. A., Megaloikonomos, P. D., Dimopoulos, L., Mitsiokapa, E., Koulouvaris, P., and Mavrogenis, A. F. 2018. "Thromboembolism Prophylaxis in Orthopaedics: An Update." EFORT Open Reviews 3 (4):136-48.

[2] Falck-Ytter, Y., Francis, C., Johanson, N. et al. 2012. "Prevention of VTE in Orthopedic Surgery Patients: 245 Antithrombotic Therapy and Prevention of Thrombosis, 9th Edition: American College of Chest 246 Physicians Evidence-based Clinical Practice Guidelines." Chest 141: e278S-e325S.

[3] Carter, D., Atay, J., Fanikos, J., and Resseguie, A. 2014. "Evaluation of Different Strategies for Prevention of 248 Venous Thromboembolism in Elective Orthopedic Surgery." J Pharmacy Pharmacology 249 (6).

[4] American Academy of Orthopaedic Surgeons. 2011. Preventing Venous Thromboembolic Disease in Patients Undergoing Elective Hip and Knee Arthroplasty. Evidence based guidelines and evidence report. September 


\section{Elective Orthopedic Surgery}

24 ,

2011. http://www.aaos.org/research/guidelines/VTE/VTE_full_ guideline.pdf (accessed 2018 Jun 08).

[5] Eriksson, B. I., Dahl, O. E., Rosencher, N., Clemens, A., Hantel, S., Feuring, M., et al. 2015. "Oral Dabigatran Etexilate Versus Enoxaparin for Venous Thromboembolism Prevention after Total Hip Arthroplasty: Pooled Analysis of Two Phase 3 Randomized Trials (Report)." Thrombosis Journal 13 (35).

[6] Fuji, T., Fujita, S., Kawai, Y., Nakamura, M., Kimura, T., Fukuzawa, M., et al. 2015. "Efficacy and Safety of Edoxaban versus Enoxaparin for the Prevention of Venous Thromboembolism Following Total Hip Arthroplasty: STARS J-V.” Thromb J 13: 27.

[7] Fuji, T., Wang, C., Fujita, S., Kawai, Y., Nakamura, M., Kimura, T., et al. 2014. "Safety and Efficacy of Edoxaban, an Oral Factor Xa Inhibitor, versus Enoxaparin for Thromboprophylaxis after Total Knee Arthroplasty: The STARS E-3 Trial.” Thromb Res 134 (6): 1198-204.

[8] Anderson, D. R., Dunbar, M., Murnaghan, J., Kahn, S. R., Gross, P., Forsythe, M., et al. 2018. "Aspirin or Rivaroxaban for VTE Prophylaxis after Hip or Knee Arthroplasty." N Engl J Med 378 (8): 699-707.

[9] Lassen, M. R., Ageno, W., Borris, L. C., Lieberman, J. R., Rosencher, N., Bandel, T. J., et al. 2008. "Rivaroxaban versus Enoxaparin for Thromboprophylaxis after Total Knee Arthroplasty.” N Engl J Med 358 (26): 2776-86.

[10] Lassen, M. R., Gallus, A., Raskob, G. E., Pineo, G., Chen,
D., and Ramirez, L. M. 2010. “Apixaban versus Enoxaparin for Thromboprophylaxis after Hip Replacement." N Engl J Med 363 (26): 2487-98.

[11] Lassen, M. R., Raskob, G. E., Gallus, A., Pineo, G., Chen, D., and Hornick, P. 2010. "Apixaban versus Enoxaparin for Thromboprophylaxis after Knee Replacement (ADVANCE-2): A Randomised Double-Blind Trial." The Lancet 375 (9717): 807-15.

[12] Turpie, A. G., Lassen, M. R., Davidson, B. L., Bauer, K. A., Gent, M., Kwong, L. M., et al. 2009. "Rivaroxaban versus Enoxaparin for Thromboprophylaxis after Total Knee Arthroplasty (RECORD4): A Randomised Trial.” The Lancet 373 (9676): 1673-80.

[13] Anderson, D. R., Dunbar, M. J., Bohm, E. R., Belzile, E., Kahn, S. R., Zukor, D., et al. 2013. "Aspirin versus Low-Molecular-Weight Heparin for Extended Venous Thromboembolism Prophylaxis after Total Hip Arthroplasty: A Randomized Trial.” Ann Intern Med 158 (11): 800 .

[14] Stewart, D. W., and Freshour, J. E. 2013. "Aspirin for the Prophylaxis of Venous Thromboembolic Events in Orthopedic Surgery Patients: A Comparison of the AAOS and ACCP Guidelines with Review of the Evidence." Ann Pharmacother 47 (1): 63-74.

[15] Connolly, S. J., Milling, T. J. J., Eikelboom, J. W., Gibson, C. M., Curnutte, J. T., Gold, A., et al. 2016. "Andexanet Alfa for Acute Major Bleeding Associated with Factor Xa Inhibitors." N Engl J Med 375 (12): 1131. 